\title{
The Romanian PhD Students at CERN: The Bologna Process and Beyond
}

\author{
Alexandru Nicolin and Florin Buzatu
}

\section{Introduction}

The modifications to the Romanian higher-education education system that followed the national implementation of the Bologna process have had a domain-specific impact within the large Romanian academic community. While the legislative changes affected equally all disciplines (for example, the transition to the three-cycle structure Bachelor-Master-PhD), the efficiency of the quality assurance policies and mechanisms depended strongly on the maturity of the academic community in charge of their implementation. The Romanian physics community, in particular, is among the most internationalized ones, with long-standing scientific collaborations both at European level and worldwide, and a research output of the highest quality (Popescu 2000). This, in turn, ensured the quality of the physics higher-education system which, for the past six decades, has been competitive worldwide, independent of political changes. The Magurele Physics Platform which emerged in early 1950s has played a paramount role in consolidating the aforementioned international stature of the Romanian physics community through continuous forefront scientific research. To give just two examples, one year after Theodore Maiman invented the first working laser in 1960, Ion Agarbiceanu created the first gas laser in Romania, while the Extreme Light Interaction-Nuclear Physics (ELI-NP) experimental facility, which is currently under construction in Magurele, will provide "magnificent new opportunities to study the fundamental processes unfolded during light-matter interaction" using the most intense lasers world-wide, and will foster an unprecedented interdisciplinary research plan which

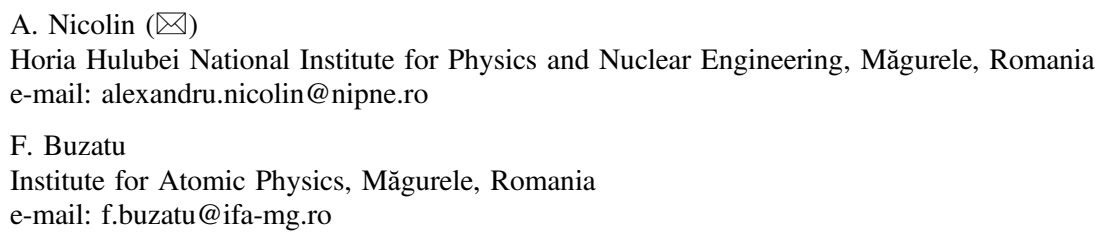


addresses "frontier fundamental physics, new nuclear physics and astrophysics as well as applications in nuclear materials, radioactive waste management, material science and life sciences" (see Habs et al. 2011 for a detailed discussion). To put it in a nutshell, the ELI-NP reflects the philosophy of the H2020 programme that "excellence needs interdisciplinarity and relevance" (as summarized by the Science Europe Position Statement on H2020, December 2012), which received less emphasis in the previous Framework Programmes.

The Magurele Physics Platform now hosts the Faculty of Physics of University of Bucharest, the Horia Hulubei National Institute of Physics and Nuclear Engineering, the National Institute for Laser, Plasma and Radiation Physics, the National Institute of Material Physics, the National Institute for Earth Physics, the National Research and Development Institute for Optoelectronics and, finally, the Institute of Space Sciences. According to the current regulations all PhD students are enrolled in the Doctoral School of a University, but they are free to carry out their research plan in a university or in a research institute under a $\mathrm{PhD}$ Coordinator who is affiliated to a Physics Doctoral School. In this contribution, we show that the PhD students who worked at CERN have experienced firsthand the values of two distinct academic cultures, and discuss in some detail the means through which we can foster a research environment that is closer to the values of the Horizon2020 (H2020) programme and thereby increase the quality of doctoral programmes.

\section{The RO-CERN Programme}

The history of CERN is well known so we will not cover it here (see, for instance, Kowarski 1961). We state, however, that roughly half of the particle physicists worldwide are working at CERN, and that they represent more than 500 institutions (universities and research institutes) and 80 nationalities. Romania's participation in CERN activities started in 1991, but the Law that effectively established the cooperation dates from 2010 (see Monitor Oficial al Romaniei number 728, November 2010). During the period 2010-2013, Romania participated in seven of the programs running at CERN, namely ALICE (A Large Ion Collider Experiment), ATLAS (A Toroidal Large Hadron Collider Apparatus), LHCb (Large Hadron Collider beauty), WLCG (Worldwide Large Hadron Collider Computing Grid), DIRAC (DImeson Relativistic Atom Complex), n_TOF (neutron time-of-flight), and ISOLDE (Isotope Separator on Line). The participation takes place through the Horia Hulubei National Institute of Physics and Nuclear Engineering (IFIN-HH-http:// www.nipne.ro) and the Institute of Space Sciences (ISS - http://www.spacescience. ro). The collaboration was later extended to include the POLITEHNICA University of Bucharest, the Alexandru Ioan Cuza University of Iasi, the West University of Timisoara, and the National Institute for Research and Development of Isotopic and Molecular Technologies, Cluj-Napoca.

Romania's contribution amounted to more than 70 physicists and engineers working on the previously mentioned projects. The team consisted of both 
Table 1 Scientific output of the Romanian team working at CERN

\begin{tabular}{l|c|c|c|c}
\hline & 2010 & 2011 & 2012 & 2013 \\
\hline Granted patents & 0 & 2 & 0 & 0 \\
\hline Access at technologies, equipment, databases, etc. & 13 & 13 & 13 & 13 \\
\hline Publications & 38 & 105 & 231 & 220 \\
\hline Communications at conferences & 108 & 166 & 183 & 126 \\
\hline
\end{tabular}

experienced scientists and graduate students, 10 of which have defended their $\mathrm{PhD}$ theses and 4 their MSc theses during that period. Moreover, the RO-CERN programme has supported $3 \mathrm{PhD}$ students whose main fields of research are not connected to CERN, who were, however, involved in the support activities for the grid computing infrastructure. The research activities of the graduate students took place both at CERN and at the premises of IFIN-HH and ISS, with research stages at CERN ranging from a couple of weeks to a few months.

The scientific output of the Romanian team working at CERN during 2010-2013 is summarized in Table 1 , where one immediately notices the large number of publications and conference communications. While the two patents obtained in 2011 (one of which is focused on Detectors for time-of-flight measurements for charged particles and another one on Detectors for measuring transition radiation) have no graduate students as co-authors, due to the advanced engineering nature of the patents, almost all papers produced within the CERN projects have graduate students as co-authors. The list of journals is impressive and contains prestigious physics journals such as Physical Review Letters, Physical Review D, etc. Moreover, graduate students have contributed significantly to the creation of new computing centres within the Romanian Tier-2 Federation, the most important one being that of the scientists of IFIN-HH who work at ALICE, which amounts to approximately 5000 computer cores and a 4 PB of storage. The efficiency of the computing centres is reflected by the fact that the Romanian Tier-2 national centre ranked in the first 10 of the 34 states that participated at WLCG.

\subsection{Interdisciplinary Research Teams}

The scientific exposure of the graduate students working in the RO-CERN programme covers a wide set of disciplines which includes experimental, computational and theoretical physics, applied mathematics, computer science, scientific engineering, as well as digital and analogue electronics. Buzatu (2011) discusses in detail the contribution of the Romanian scientists at CERN, experiment by experiment, and shows that it spans a wide range of topics which includes: operating the experiments (in shifts, jointly with the other scientists), theoretical high-energy physics calculations, designing and constructing particle detectors, algorithm analysis and comparison, comparing theoretical results obtained by numerical means and 
experimental data, software development for the analysis and interpretation of data, optimizing software implementation (to reduce the computational load), day-to-day running and periodic upgrading of Data and Computing Centres, etc. This broad scientific exposure reflects the complexity of the research activities in which the Romanian groups at CERN are involved, and conveys to the graduate students the clear message that forefront scientific physics research transcends the traditional domanial boundaries and relies heavily on interdisciplinary exchanges. Interdisciplinarity is a key feature of all Romanian groups working at CERN which consist both of scientists with solid physics backgrounds, as well as research and development engineers, computer scientists (both hardware and software oriented), electronic engineers, and various types of technicians (see Buzatu 2011) for a detailed discussion on the contributions of the Romanian teams). This broad scientific milieu should be contrasted with that commonly seen in the Physics Doctoral Schools of Romanian universities which consist mainly of physicists, with considerably fewer scientists of different backgrounds. This difference in the structure of the research groups reflects, on one hand, the mission of the institutions in which the graduate students are active (IFIN-HH, to give just one example, is an institute dedicated to "physics and nuclear engineering" [our italics]), while on the other hand, it reflects how the actual research is performed. To put it simple (and thereby leave out many details), university research relies on a number of small research groups whose interests cover a wide set of topics, while the Romanian national research and development institutes focus on a single research field (such as nuclear physics, laser physics, material sciences, etc.) and address coherently a series of related theoretical, computational and experimental problems, as to significantly increase our understanding of the research field in focus. While research in theoretical physics usually excludes interdisciplinary exchanges, with the possible exception of scientific programming and applied mathematics, the computational and the experimental research require considerable expertise in numerous scientific and engineering braches.

The differences between the research landscapes of the two types of institutions discussed above (i.e., national research and development institutes and universities) are also reflected by the competence-based research funding mechanisms used to finance their graduate students. University-based graduate students were supported either through individual research grants (most of them funded using the Structural Funds in the Sectoral Operational Programme Human Resources Development), or through small-scale projects, usually involving less than a dozen scientists, funded by the Romanian Executive Agency for Higher Education, Research, Development and Innovation Funding (UEFISCDI). In addition to these financing mechanisms, the physics graduate students employed at the research institutes which are involved in the RO-CERN programme were also supported through the research projects financed by the Institute of Atomic Physics (IFA). These projects were considerably larger than those coordinated by UEFISCDI and were subject to less significant budgetary cuts which, in turn, offered PhD students working at CERN greater stability. 


\subsection{Multiculturalism and Internationalization}

Multiculturalism is usually irrelevant in discussions concerning the training of physics graduate students in Romania, but this is not the case for the students working at CERN. CERN is widely considered the first European Institution and among the documents which preceded its foundation is the communication of Louis de Broglie at the 1949 European Cultural Conference in Lausanne (read by Raoul Dautry) in which he spoke of "the creation of a laboratory or institution where it would be possible to do scientific work, but somehow beyond the framework of the different participating States", such that this new laboratory or institution can "undertake tasks, which, by virtue of their size and cost, were beyond the scope of individual countries" [the original French version of the letter appears in Kowarski (1961)]. The final paragraph of the letter emphasizes that "this form of collaboration has to be one of the most immediate objectives of those who take upon themselves to bring together the European nations and to ensure the collaboration of the underlying set of values for the progress of civilization." Decades later, the research output of the Romanian scientists working at CERN (see Table 1) as well as the numerous CERN events organized in Romania (such as summer schools, info days for high-school students, etc.) show that these ideas were embraced not only in Western but also in Eastern Europe. In Romania's case, which joined CERN in 2010, the active agents of this European kinship were the graduate students who participated actively in the local experiments. At this point it is worth mentioning that the same vision which led to the creation of CERN was shared, though at a small scale, by the scientists and political figures in Eastern Europe and the states of the former Union of Soviet Socialist Republics who formed in 1956 the Joint Institute for Nuclear Research in Dubna.

While most discussions in Romania about the Bologna Process are focused on insuring similar quality standards and an increased coherence of the national higher education systems, the principles and the spirit of the Bologna Process are best embodied in the graduate students working at CERN. The research plans of these students are congruous with those of their peers, their research philosophy is very similar, they benefit from the same type of courses and training seminars, the working conditions are identical, with no differences between students from different nations, and the same quality and ethical standards are followed by everybody. The long lists of authors of the papers coming out of CERN (in which the contributors are arranged alphabetically) reflect this unique scientific melting-pot, while the recent experiments which proved the existence of the Higgs boson that led to 2013 Physics Nobel Prize show unequivocally that the research is of the highest international level. Moreover, Romania's substantial involvement at CERN shows that the physics higher education system is compatible with its European siblings, and that Romanian graduate students can integrate in the most challenging research environments. The key ingredient responsible for the aforementioned compatibility is the substantial internalization of the Romanian physics community, which is reflected in numerous large-scale international collaborations (see Buzatu 2011) 
and, most importantly, an uninterrupted intellectual tradition (Frangopol 2012). This tradition goes back to Horia Hulubei, an outstanding atomic scientist who worked with Jean Perrin at the Sorbonne for his $\mathrm{PhD}$ thesis and the founding father of the Magurele Physics Platform, and is largely responsible for the strict adherence to the academic customs, norms and criteria widely accepted internationally (see Frangopol 2012). It is noteworthy to add that physics research was never influenced directly by political changes and reached a level of stability which, in turn, allowed Romanian physicists to follow their research interests with a higher degree of freedom than that of their colleagues working, for instance, in social sciences and humanities. Naturally, the general decisions concerning the access to scientific literature, attendance at international meetings and alike affected the physics community during the communist period just as they affected any other professional community, but no decision detrimental to the development of the physics community was directly targeted at it.

\subsection{Personal Research Contributions}

One of the most delicate aspects concerning the research plans of Romanian PhD students working at CERN refers to the actual personal contribution to the numerous publications. The paternity of scientific results is always problematic in the case of large collaborations, and this is why the Romanian $\mathrm{PhD}$ regulations for awarding $\mathrm{PhD}$ diplomas in physics emphasize the importance of the articles in which the PhD student is the first author (provided, of course, the listing of author is not done alphabetically). One tradition in the physics community is that (with the notable exception of large collaborations mentioned explicitly in the text) the order in which the authors appear reflects the contribution to the article, with the first author having the most important contribution, and the last one usually ensuring the coordination of the team. The supporters of this tradition usually advocate the idea that for the papers coming out of the CERN collaboration authorship should be viewed more as a form of credit for scientific service, rather than a clear indication of specific contributions. While this tradition is not widely accepted, with prestigious journals such as the Physical Review series stating explicitly that "the names of authors may be listed in any order in the byline between the title and abstract" and other journals, such as Nature, requiring a short description of the contribution of each author, its inclusion in the Romanian $\mathrm{PhD}$ regulations catalyzed the production of papers outside the main flux of the CERN collaboration. These papers (usually in the form of articles in local peer-reviewed journals and conference proceeding) ensured that the $\mathrm{PhD}$ theses of the Romanian graduate students at CERN also contain research results which can be clearly attributed to their authors.

In fact, this problem is quite general, as many national-level regulations concerning the eligibility to become Associate Professor, Full Professor, and $\mathrm{PhD}$ Coordinator, as well as the eligibility criteria for coordinating national research projects, and many internal criteria used by Romanian universities and institutes to 
assess scientific output are very sensitive on the number and order of authors. All these regulations draw from the Minimal Criteria for Associate Professor, Full Professor, and PhD Coordinator established as part of the by-laws which followed the 2011 National Education Law. Small details aside, the criteria currently in place assess the quality of the research output using the Article Impact Score as the main indicator, unlike the older ones which relied on the Impact Factor. The assessment focuses both on the bulk of the scientific output (using a normalization that accounts for the number of authors of each paper) and, more importantly, on those papers for which a given scientist is first (or corresponding) author. This last component of the assessment is problematic for scientists working in high-energy physics where such personal contributions are not particularly important, and is detrimental to the development of the high-energy physics community.

In the context of the previous remarks we would like to emphasize the following aspect: the criteria used to evaluate the scientific output of PhD students (both with respect to quality and with respect to quantity) can generate considerable imbalances between the various branches of physics if the peculiarities of each particular branch are not properly reflected in the criteria. As the Bologna Process standardized the three- or four-years doctoral programmes, while the particularities of the current physics research areas are vastly different, it is important that the final assessment process of the scientific achievements accounts correctly the specificities of each research area. Moreover, wherever possible, it is desirable to complement scientometrics indicators with expert peer-review, such that the relatively short PhD stage is best used to maximize the scientific output relevant for that specific research area, and not for the mechanical fulfilment of scientific criteria of disputable relevance. In other words, the $\mathrm{PhD}$ students should fulfil the specific requirements of their projects and pay lesser attention to generic national criteria (currently based on scientometrics) which do not capture the specificities of all fields. After all, scientometrics assessments are always precise, but not always relevant, as "not everything that counts can be counted, and not everything that can be counted counts" (Albert Einstein). This is particularly relevant for graduate students, such as the Romanian PhD students working at CERN, who perform their actual research in an academic culture which values diversity and interdisciplinarity, but are finally assessed outside that academic culture using a more stringent (actually narrower) set of rules. In fact, the tendency to always quantify the output of $\mathrm{PhD}$ students can be detrimental to the creativity that leads to major scientific breakthroughs, and induces a level of uniformity that does not foster scientific excellence and innovation. Moreover, the regulations on the eligibility criteria for Associate Professor which do not consider the particularities of the research carried out at CERN can reduce the appeal of a future academic carrier for the PhD students working at CERN. It is, of course, desirable to have stable criteria for awarding $\mathrm{PhD}$ diplomas and being eligible to Associate Professor, such that all scientists have the same professional opportunities and the dynamics of the community is not perturbed by arbitrary factors.

One section of the 1999 Bologna declaration states that "we must in particular look at the objective of increasing the international competitiveness of the European 
system of higher education. The vitality and efficiency of any civilisation can be measured by the appeal that its culture has for other countries. We need to ensure that the European higher education system acquires a world-wide degree of attraction equal to our extraordinary cultural and scientific traditions." The appeal and the attraction mentioned above can only be achieved in a stable academic system which welcomes diversity, and acknowledges that forefront research is usually interdisciplinary and cannot be assessed using the classical evaluation methods. The aforementioned stability implies that the dynamics of an academic community is governed from within the community without disruptive external factors (such as massive budgetary cuts and constant changes of the criteria used to evaluate the quality and the impact of the scientific output), while interdisciplinarity implies a broad view of the research landscape. To give just one example, the computer codes used to analyze the experimental data obtained at CERN are a crucial ingredient of the research plan, but are not acknowledged by any of the current criteria. These codes require a thorough understanding of the underlying physics, as well as substantial knowledge of numerical analysis, applied mathematics, parallel programming, etc., and usually amount to many months (sometimes years) of collaborative hard work. Assessing such a scientific endeavour within the classical paradigm that physicists produce mainly books, articles and patents is clearly impossible, and one has to rely on expert peer review.

The previous discussion is also pertinent for the world-class ELI-NP experimental facility which is currently built on the Magurele Physics Platform. As most of the experiments envisaged to take place at ELI-NP rely on interdisciplinary research teams which will deal with numerous difficult technical and scientific problems, some (if not most) of which have not yet been identified, it is important that the graduate students employed at the facility will receive proper recognition for their work. We emphasize that in early operational stages of the facility, the classical research output (i.e., papers) will be minimal and that most of the efforts will be focused on dealing with the inevitable experimental bottlenecks, therefore the scientific profile of the first series of graduate students at ELI-NP will be substantially different than that of their peers working, for instance, in theoretical or computational physics.

Let us also mention that this broadening of our understanding of the research landscape is crucial for the success of the H2020 programme, and that the fresh impetus on increasing the transfer of knowledge to the industry will not reach its full impact without a modification of the assessment framework (both for $\mathrm{PhD}$ students and Professors), such that experimental and functional models, prototypes and demonstrative models, innovative services, etc., are properly acknowledged as significant forms of scientific output. In the context of the Bologna Process it is very important to convey to the students the proper importance of all forms of scientific output and prepare them for the challenges ahead. The philosophy of H2020 is that of accelerating the social impact of discoveries and innovation by taking great scientific ideas from lab to market quicker than before, and the perspective on research that we pass on to the graduate students should follow this closer connection between research, innovation and industrial development. To put it 
otherwise, as part of the graduates of the third cycle of the Bologna Process will become active in the H2020 research programmes as junior scientists, it is extremely important that their education prepares them for the challenges ahead. Finally, in light of these changes, it is obvious that the education of the PhD students should be complemented with elements of entrepreneurship and intellectual property. The entrepreneurial spirit of the future generation of scientists plays a key role in short-circuiting the distance between scientific discovery and industrial applications, while a good understanding of the intellectual property rights ensures a level of recognition that goes outside the realm of purely academic merits.

\section{Conclusions}

The Romanian participation at CERN has provided unique opportunities for physics graduate students and has strengthened the scientific output of the doctoral schools, both qualitatively and quantitatively. By exposing graduate students to forefront achievements in engineering, technology and physics, these international collaborations fostered an unparalleled multidisciplinary environment that is conducive to innovative solutions to the most challenging scientific problems, as evidenced, for instance, by the two patents obtained by the ALICE Romanian team in 2011. One of the problems faced by the Romanian $\mathrm{PhD}$ students working at CERN concerns the criteria used to evaluate their work. These assessment criteria do not reflect the research philosophy of large-scale experimental facilities where the collaborative nature of the research is prominent, and emphasize the significance of personal contributions as seen from the number and ordering of authors on a given paper. Moreover, as most assessment criteria concerning $\mathrm{PhD}$ students refer solely to the number of published articles, part of their work at CERN remains un-acknowledged. Given the goal of the H2020 programme, to shorten the distance between scientific research and industrial application as to ensure the transition to a more innovative and competitive Europe, we should broaden our assessment framework such that we properly account for all forms of scientific output, and try to complement, where possible, the current scientometrics evaluations with expert peer-review. It is our experience that the prescriptive application of strict assessment standards is not fully compatible with supporting the specificities of each research field and does not catalyze creativity and innovation. To foster a creative and innovative environment, we should encourage interdisciplinarity and diversity, and always keep in mind that the core component of the doctoral training is the advancement of knowledge through original research, not for original research, an aspect which seems of second importance in the current discussion on the Bologna process. In other words, the inclusion of the $\mathrm{PhD}$ students in research activities is done to build solid research competences and clear transferable skills that are the basis for the future career of the PhD students outside of the academic sector. 
Open Access This chapter is distributed under the terms of the Creative Commons Attribution Noncommercial License, which permits any noncommercial use, distribution, and reproduction in any medium, provided the original author(s) and source are credited.

\section{References}

Buzatu, F. (2011). The assessment of the Romanian research potential in physics and the elaboration of the international cooperation strategy. Paper presented at the Romania's participation strategy at large physics international collaborations. http://www.ifa-mg.ro/esfro/ docs/etape/Etapa5/Raport_V.pdf

Frangopol, P. T. (2012). Horia Hulubei. Revista de Politica Stiintei si Scientometrie-Serie Noua, 1(3), 178-183.

Habs, D., Tajima, T., \& Zamfir, N. V. (2011). Extreme light infrastructure-nuclear physics (ELI-NP): New horizons for photon physics in Europe. Nuclear Physics News, 21(1), 23-29. Kowarski, L. (1961). An account of the origin and the beginnings of CERN. Geneve: CERN. Popescu, M. (2000). Archaeus' whisper, part I (from the history of physics at Magurele platform). Bucharest: Horia Hulubei Publishing House. 